\title{
Análisis del rendimiento académico de los estudiantes de Ingeniería de Sistemas, posibilidades de deserción y propuestas para su retención
}

\author{
Analysis of the academic performance of Systems Engineering students, \\ desertion possibilities and proposals for retention
}

Norka Bedregal-Alpaca $^{1 *} \quad$ Doris Tupacyupanqui-Jaén ${ }^{1} \quad$ Víctor Cornejo-Aparicio $^{1}$

Recibido 15 de julio de 2020, aceptado 1 de septiembre de 2020

Received: July 15, 2020 Accepted: September 1, 2020

\begin{abstract}
RESUMEN
Los problemas de rezago en los estudios y deserción afectan a las instituciones educativas, a los estudiantes que los padecen y a sus familias, de allí la importancia de estudiarlos.

En este trabajo, se analiza el rendimiento académico de las cohortes 2011-2016 de la Escuela Profesional de Ingeniería de Sistemas de una universidad pública, para ello se cuenta con información de 976 estudiantes: puntaje de ingreso a la universidad, calificaciones en las asignaturas y alguna data personal. Se describe estadísticamente el rendimiento académico general y el del primer año de estudios. Se calculan el promedio ponderado ajustado y los índices de rendimiento exógeno, endógeno y total. Con todo este conjunto de variables, utilizando un aplicativo propio y un aplicativo comercial, se aplican técnicas de minería de datos para encontrar patrones que describen el comportamiento académico estudiantil; para ello se emplea la metodología CRISP-DM.

$\mathrm{Al}$ aplicar técnicas de clasificación: redes neuronales y árboles de decisión se encuentra que las variables más influyentes son el índice de rendimiento exógeno y la relación de créditos aprobados en relación a los créditos que en teoría debieron aprobar.

Como consecuencia se proponen algunas estrategias que adecuadamente implementadas podrían disminuir los problemas estudiados.

Se concluye que al analizar el rendimiento académico de un estudiante no basta con las calificaciones obtenidas, se debe considerar su comportamiento académico, su rendimiento en relación a su cohorte y el ritmo de avance en la aprobación de las asignaturas. Es así que las técnicas empleadas permiten identificar oportunamente a los estudiantes en riesgo académico.
\end{abstract}

Palabras clave: Rendimiento académico, deserción estudiantil, reprobación académica, rezago estudiantil, minería de datos, técnicas de clasificación.

\section{ABSTRACT}

Problems with late studies and desertion affect educational institutions, students who have them, and their families, hence the importance of studying them.

In this work, we analyze the academic performance of the 2011-2016 cohorts of the Professional School of Systems Engineering of a public university, for this we have information from 976 students: university admission score, subjects' qualifications and some personal data.

The overall academic performance and the first year of study are statistically described. The adjusted weighted average and exogenous, endogenous, and total performance rates are calculated. With this set

\footnotetext{
1 Universidad Nacional de San Agustín de Arequipa. Departamento Académico de Ingeniería de Sistemas e Informática. Arequipa, Perú.E-mail: nbedregal@unsa.edu.pe; dtupacyupanqui@unsa.edu.pe; vcornejo@unsa.edu.pe

* Autor de correspondencia: nbedregal@unsa.edu.pe
} 
of variables, using a proprietary app and a commercial app, data mining techniques are applied to find patterns that describe student academic behavior.

By applying classification techniques: Neural networks and decision trees, it is found that the most influential variables are the exogenous performance rate and the ratio of approved credits in relation to the credits that in theory had to be approved; for this the CRISP-DM methodology is used.

As a result, some strategies are proposed that could decrease the studied problems.

It is concluded that when analyzing a student's academic performance, the qualifications obtained are not sufficient, their academic behavior, their performance in relation to their cohort and the pace of progress in the approval of the subjects should be considered. Thus, the techniques used allow students at academic risk to be identified in a timely manner.

Keywords: Academic performance, student desertion, academic reprobation, student lag, data mining, classification techniques.

\section{INTRODUCCIÓN}

La educación es fundamental para el desarrollo y el bienestar de una sociedad, por tanto, los estudiantes son el activo fundamental de cualquier institución educativa. El desarrollo social y económico de un país está directamente relacionado con el rendimiento académico de sus estudiantes [1].

L análisis del rendimiento académico debe considerar problemas relacionados con el "retraso en los estudios" y la "deserción", problemas que son mundialmente reconocidos y que significan una pérdida de capital humano y financiero, pues impactan negativamente en las instituciones, en los sistemas de educación superior y, principalmente, en los estudiantes que los padecen y en sus familias. Se suma a esta problemática el hecho de que la concepción de esos términos no es única.

En la década pasada, el Instituto Internacional para la Educación Superior en América Latina y el Caribe (IESALC) propuso un Proyecto Regional sobre Deserción y Repitencia en la Educación Superior en América Latina y el Caribe, cuyo objetivo era determinar la magnitud de la deserción y la repitencia en los países de la región en el ámbito de sus sistemas de educación superior, analizar sus causas, sus implicaciones y proponer medidas remediales [2].

El Banco Mundial a través de un estudio realizado por [3] afirma "En América Latina y El Caribe la mitad de la población de 25-29 años de edad que comenzaron la educación superior en algún momento no finalizaron sus estudios, sea porque aún están estudiando o porque desertaron"; así mismo, ha estimado que alrededor del $42 \%$ de estudiantes que inician sus estudios universitarios abandonan el sistema de educación superior y que más del $30 \%$ lo hacen al final del primer año.

Para los países y las universidades, el atraso en los estudios y la deserción estudiantil son los principales obstáculos en el camino a garantizar el acceso igualitario y la permanencia en la educación superior. Por otra parte, una de las dimensiones más importantes en los procesos formativos es el rendimiento académico del estudiante, ya que se le considera un indicador de éxito o fracaso del estudiante y por ello es el aspecto principal que se estudia al analizar los resultados del proceso educativo. En América Latina, en particular, estas problemáticas son álgidas y deben estudiarse considerando el contexto socio-económico y las características del sistema educativo de cada país.

En Perú, las cifras resultan mucho más alarmantes si se considera que la educación es la mejor herramienta para el desarrollo del país. La investigación realizada por el Banco Mundial, a finales de 2018, reveló que cerca del $30 \%$ de los universitarios peruanos abandonan su carrera por diferentes motivos, siendo mayor la deserción en el caso de las universidades privadas. Según Penta Analytics, en el 2017, el $27 \%$ de los ingresantes a una universidad privada abandonan su carrera en el primer año de estudios. Según el Instituto Nacional de Estadística e Informática (INEI), de cada diez estudiantes que ingresan a las universidades, solo cinco concluyen sus estudios en el tiempo predeterminado. Otro estudio elaborado por la consultora Flanqueo, 
señala que por lo menos el $30 \%$ de los estudiantes empieza a retrasarse principalmente en el primer semestre, llegando a retrasarse muchos ciclos o incluso a abandonar la carrera.

Surge entonces la interrogante ¿es posible evitar que los jóvenes se retrasen en los estudios o abandonen la universidad? En el contexto descrito y con objeto de responder a esa interrogante, en la Escuela Profesional de Ingeniería de Sistemas de la Universidad Nacional de San Agustín (EPIS-UNSA) se ha trabajado en un proyecto de investigación para dar respuesta a un conjunto de interrogantes derivadas: ¿El rendimiento académico estudiantil está relacionado con la deserción? ¿En qué ciclo académico estas problemáticas tienen mayor incidencia? ¿Qué características tiene el rendimiento académico de los estudiantes? ¿Qué estrategias administrativas y académicas contribuirán a prevenir el retraso o la deserción de los estudiantes?

Con los resultados de esta investigación se espera lograr que los estudiantes mejoren su desempeño académico y logren finalizar sus estudios.

El estudio realizado no pretende ser concluyente, sólo constituye un aporte al estudio del rendimiento académico universitario en la UNSA.

\section{MARCO CONCEPTUAL}

\section{Rendimiento académico (RA)}

En términos generales el rendimiento académico está relacionado con el éxito o eficiencia del proceso educativo y se determina tomando en cuenta variables cualitativas y/o cuantitativas.

Diversos autores han tratado de definir el rendimiento académico, por lo que en la literatura se encuentra: "es el resultado del proceso de aprendizaje académico en el que influyen variables sociales, personales y escolares y sus interrelaciones" [4]. Según [5] el RA es un resultado del aprendizaje, suscitado por la intervención pedagógica del profesor y producido en el alumno.

Bloom (citado en [6] ) afirma "es lo necesario para que el alumno sea capaz de llevar a la práctica sus conocimientos, que pueda aplicar la información adquirida a la solución de problemas; en pocas palabras, es el resultado del trabajo escolar".
Cuando se evalúa el rendimiento académico con objeto de mejorarlo, se parte por analizar los factores que por los que se ve influenciado. Existen varios estudios sobre los factores que influyen en el rendimiento académico de los estudiantes [5, 7-9], concluyen que los factores más comunes se distribuyen en dos vertientes: socioeconómica y académica. Desde la vertiente académica influye la formación base del estudiante, las características del programa de estudio, las metodologías de enseñanza-aprendizaje utilizadas, la masificación de la enseñanza, el nivel de pensamiento formal de los estudiantes, los hábitos de estudio, entre otros.

\section{Deserción estudiantil}

Se han dado varios acercamientos al concepto de deserción estudiantil, muchos de ellos se ven influenciados por el tipo de investigación en el que se analiza el concepto. En [10] se define la deserción como "un estado en el que el estudiante se enfrenta a una situación en la que no logra concluir su proyecto educativo".

En [5] se considera como desertor al estudiante que, habiendo estado matriculado en una institución universitaria, durante tres semestres académicos consecutivos no vuelve a registrar matrícula. Otros estudios postulan que la deserción es el resultado de la comparación cuantitativa entre la matrícula inicial y la cantidad de estudiantes graduados del último año.

Diversos estudios han ensayado diferentes factores para explicar la deserción: mala asesoría vocacional, carencia de conocimientos base, desempeño académico, problemas económicos, presión dentro del núcleo familiar y el estrés de mantenerse competitivo dentro de la carrera, entre otros [11-13].

\section{Rezago estudiantil}

Cuando se hace referencia al rezago estudiantil, se alude a los estudiantes que no logran los objetivos educativos y por tanto reprueban una o más asignaturas. La reiteración de estos casos deriva en el fracaso en los estudios y muchas veces en el abandono de los mismos por parte de los estudiantes.

Indicadores de medida del rendimiento académico Según [14] los indicadores más utilizados para medir el rendimiento académico son:

- Calificaciones: Representan una medición cuantitativa o cualitativa del rendimiento. 
- Pruebas objetivas: Dado que las respuestas son precisas tienen ventaja en el grado de objetividad (no interviene la subjetividad del profesor).

- Número de asignaturas aprobadas: Sirve para determinar el rendimiento académico como medida de éxito/fracaso. Un estudiante tendrá éxito si aprueba la mayoría de las asignaturas en las que se matriculó en un periodo académico, si aprueba menos de la mitad se podría decir que tuvo un bajo desempeño académico y si no aprueba ninguna se diría que el estudiante fracasó académicamente.

- Número de Créditos acumulados: Análogo al indicador anterior, identifica el avance de un estudiante al comparar el número de créditos acumulados durante un periodo académico con el número de créditos que, de acuerdo al plan de estudios, debió acumular en el tiempo programado.

\section{Tipo de tratamiento de las calificaciones}

Existen diferentes formas de calcular el promedio de las calificaciones:

- Promedio aritmético (PA): suma de todas las calificaciones finales dividida por el número de asignaturas que cursó el estudiante. (Deficiencia: no considera la cantidad de materias reprobadas durante la carrera)

- Promedio ponderado(PP): se obtiene al dividir la suma ponderada (créditos de las materias por la calificación final) entre el total de créditos cursados. (Deficiencia: no considera la cantidad de materias reprobadas durante la carrera)

- Promedio ponderado ajustado (PPA): se obtiene al dividir la suma ajustada (créditos por promedio de las calificaciones) entre el total de los créditos cursados por el alumno durante su carrera; la calificación final de cada materia es el promedio de las oportunidades que haya tenido para pasarla.

\section{Proceso de extracción de conocimiento (Knowledge Discovery in Databases (KDD))}

La extracción de conocimiento a partir de datos es el proceso desarrollado para descubrir conocimientos interesantes, como patrones, asociaciones, cambios, anomalías y estructuras significativas a partir de grandes cantidades de datos almacenadas en bases de datos, Data-Warehouses o cualquier otro medio de almacenamiento de información.
KDD es el proceso que se encarga de preparar los datos e interpretar los resultados obtenidos, para dar así significado a los patrones encontrados. Involucra un proceso iterativo e interactivo de búsqueda de modelos, patrones o parámetros. Los patrones descubiertos serán válidos y novedosos para el sistema (para el usuario siempre que sea posible) y potencialmente útiles.

\section{CONTEXTO}

En Perú existen 142 universidades, entre ellas la gran mayoría (64\%) son privadas. La Universidad Nacional de San Agustín de Arequipa (UNSA), es la universidad pública más grande de la región sur del país lo que le impregna un sello distintivo y características particulares. Según cifras de la Superintendencia Nacional de Educación Superior Universitaria, se ubica como la segunda universidad, a nivel nacional, con mayor número de postulantes; cada año aumenta el número de postulantes y paradójicamente el proceso de selección sigue siendo el tradicional examen de opción múltiple, sin tener en cuenta que los ingresantes están cada vez más expuestos a múltiples y diversos retos en el campo académico, económico y social [15].

Los estudiantes que ingresan a la UNSA, en términos generales, no lo hacen en las mejores condiciones académicas y socioeconómicas, por lo que, en los primeros semestres muchos rezagan o abandonan sus estudios. La repitencia y la deserción son, por tanto, problemas presentes en todas las escuelas profesionales, sin embargo, en las ingenierías estos problemas tienen mayor incidencia en las asignaturas de ciencias básicas: matemática, física, química.

La investigación que aquí se relata se desarrolla en la Escuela Profesional de Ingeniería de Sistemas (EPISUNSA), escuela que enfrenta problemas de bajo rendimiento académico, deserción y rezago estudiantil.

Cada año ingresan a esta escuela entre 100 y 120 estudiantes, por lo que la población estudiantil varía entre 500 y 600 estudiantes.

\section{METODOLOGÍA}

\section{Tipo de investigación}

Este estudio se encuadra dentro de una metodología exploratoria cuantitativa, con enfoque en estudio de 
casos, y de tipo fenomenológico. Se contextualiza el proceso de investigación considerando temporalidad, espacio y contexto relacional.

\section{Sujetos de estudio}

La población está formada por las cohortes 20112016, involucra a todos los estudiantes que registraron matrícula en la Escuela Profesional de Ingeniería de Sistemas de la Universidad Nacional de San Agustín de Arequipa, Perú (EPIS-UNSA) entre los años 2011 y 2016.

La muestra queda conformada por los registros académicos de los estudiantes que pasaron el proceso de depuración, con lo que la muestra corresponde a los registros académicos de 976 estudiantes.

\section{Variables}

Como resultado del análisis de la literatura relacionada se puede afirmar que existe un conjunto de variables que determinan el comportamiento académico de los estudiantes: académicas, personales, socioeconómicas e institucionales. Para este trabajo se han tomado en cuenta las variables académicas y algunas variables de tipo personal que se encontró en la data. No se han considerado variables socioeconómicas por no tener acceso a esa información. Las variables institucionales no son materia de este estudio.

Al considerar la deserción como resultado del fracaso académico, las mismas variables consideradas para medir el rendimiento académico se han considerado como variables determinantes de la probabilidad de retraso en los estudios o de deserción estudiantil.

\section{Etapas}

Este estudio se desarrolla en 5 etapas generales:

1. Recolección de la información.

2. Análisis estadístico de la información.

3. Proceso de minado de datos.

4. Análisis de los resultados.

5. Propuesta de medidas remediales.

\section{Técnicas y herramientas}

Con el propósito de sustentar cualquier intento de contextualización empírica y para una primera caracterización de los estudiantes en relación a su comportamiento académico se utilizaron técnicas de estadística descriptiva. Los análisis se hicieron utilizando las funcionalidades de la hoja de cálculo EXCEL y el software IBM SPSS Statistics.
Para predecir el comportamiento académico y la deserción se utilizaron técnicas de minería de datos, bajo la metodología CRISP-DM. El proceso de KDD se realizó mediante un aplicativo desarrollado en JAVA y utilizando el software IBM SPSS Modeler.

\section{Recolección de la información y descripción de los datos}

Los datos iniciales los proporcionó el Instituto de Informática de la UNSA, dependencia que maneja toda la información digitalizada de la universidad. La data contiene 976 registros capturados en la ficha de matrícula al momento en que los estudiantes ingresan a la universidad, 534 registros recabados por la Oficina de Admisión y 26069 registros de calificaciones. La data se recabó a través de varios archivos Excel, por lo que fue necesario integrarlos a través de un código único (CUI) que identifica a cada estudiante.

Los datos se pueden agrupar en tres categorías:

- Datos personales de los estudiantes: CUI (código único de identificación) apellidos y nombres, fecha nacimiento, lugar nacimiento, sexo, tipo colegio, lugar colegio.

- Datos relativos al ingreso a la universidad: periodo, fase, puesto, nombre y apellidos, puntaje.

- Registro de calificaciones: CUI, código asignatura, nombre de asignatura, grupo, matricula, nota, condición, año, ciclo.

\section{ANÁLISIS ESTADÍSTICO DE LA INFORMACIÓN}

Un acercamiento a los factores que determinan el rendimiento académico y la deserción de los estudiantes debe partir de una caracterización estadística del comportamiento académico estudiantil. Es por ello, que en esta etapa de la investigación se propone cubrir la necesidad de actualizar datos estadísticos para obtener información cuantitativa sistematizada y ordenada con alto valor descriptivo y diagnóstico; todo ello con el propósito de sustentar cualquier intento de contextualización empírica.

El momento exploratorio permite identificar las características de la población de estudio, es así que, a partir de los datos recabados se realizó un análisis descriptivo de las variables académicas disponibles. En esta fase exploratoria la variable 
rendimiento académico, actúa como una variable proxy; es decir, una variable que se aproxima a ese concepto, pero que al no considerar los factores por los que se ve influenciada, no recoge el concepto exacto de rendimiento.

\section{Caracterización de la población de ingresantes} Además de los estudiantes que ingresan cada año, están aquellos que van rezagando asignaturas y que se van insertando en las nuevas promociones; también se presentan casos de abandonos de la carrera, por lo que la población estudiantil de la EPIS-UNSA es cercana a los 540 estudiantes. En la Tabla 1, se resumen las características de los puntajes de ingreso de cada cohorte.

En términos generales se observa que, los puntajes de ingreso siguen una tendencia no decreciente y de mayor homogeneidad. La Figura 1, presenta la información referida al tipo de colegio de procedencia de los ingresantes, es necesario mencionar que un análisis cualitativo reveló que los colegios particulares tenían reputación académica baja o muy baja. La Figura 2, describe la composición por género de las diferentes cohortes. Un análisis cuantitativo nos lleva a observar que el porcentaje de estudiantes mujeres tiende a decaer más a partir del octavo semestre, sin embargo, las causas se podrán explicar con información socioeconómica.

\section{Matrícula considerando la relación año de ingreso - año cronológico}

Un indicador de buen rendimiento académico es la matrícula y aprobación de las asignaturas correspondientes al plan de estudios vigente, en ese sentido se espera que el estudiante apruebe las asignaturas en las que se matricula cumpliendo con el avance, dentro del creditaje y el tiempo, programado en el plan de estudios. La Figura 3, resume la información que relaciona la proporción

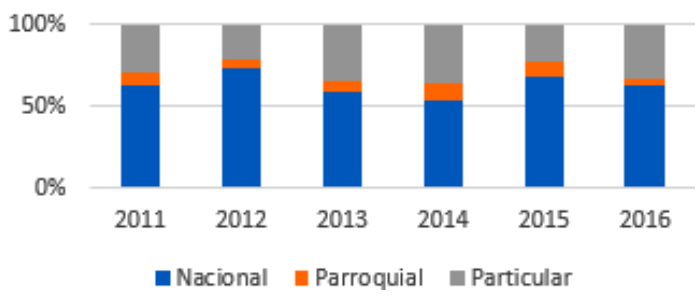

Figura 1. Porcentaje de ingresantes procedentes de los diferentes tipos de colegio.

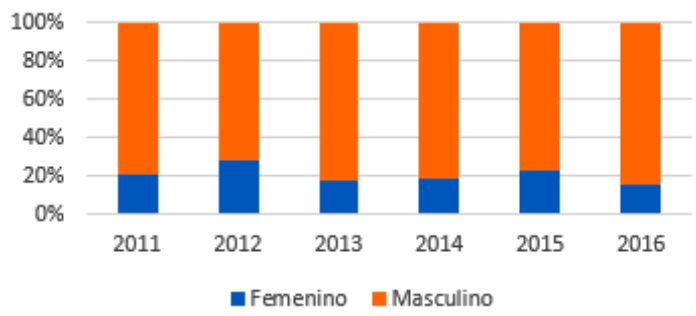

Figura 2. Distribución por género de las cohortes 2011-2016.

de estudiantes a los que les corresponde estar matriculados en cada ciclo y año (matrícula regular) y aquellos que han rezagado alguna asignatura (matrícula rezagada). Por ejemplo, en el año 2011 ciclo A, sólo el 59,8\% de los estudiantes tenía matrícula regular, los restantes estaban en condición de rezagados en por lo menos una asignatura.

\section{Estudiantes con alguna asignatura reprobada}

La Figura 4 hace un paralelo de las proporciones entre la cantidad de estudiantes con todas las asignaturas aprobadas y aquellos que tienen por lo menos una asignatura reprobada.

\section{Caracterización del primer año de estudios}

Según [16], los indicadores de carácter cuantitativo son un referente del campo de valores de variables

Tabla 1. Análisis descriptivo de los puntajes de ingreso de las cohortes 2011-2016.

\begin{tabular}{|l|c|c|c|c|c|c|}
\hline \multicolumn{1}{|c|}{ Medida } & $\mathbf{2 0 1 1}$ & $\mathbf{2 0 1 2}$ & $\mathbf{2 0 1 3}$ & $\mathbf{2 0 1 4}$ & $\mathbf{2 0 1 5}$ & $\mathbf{2 0 1 6}$ \\
\hline Media aritmética & 59,016 & 59,794 & 63,001 & 64,271 & 63,429 & 62,805 \\
\hline Desviación estándar & 7,707 & 8,853 & 8,721 & 7,131 & 8,044 & 5,456 \\
\hline Coeficiente variación & 0,131 & 0,148 & 0,138 & 0,111 & 0,127 & 0,087 \\
\hline Tamaño & 87 & 88 & 88 & 90 & 89 & 92 \\
\hline Máximo & 82,049 & 78,656 & 86,921 & 84,378 & 76,625 & 82,002 \\
\hline Mínimo & 39,416 & 41,387 & 39,425 & 43,325 & 35,014 & 52,12 \\
\hline
\end{tabular}




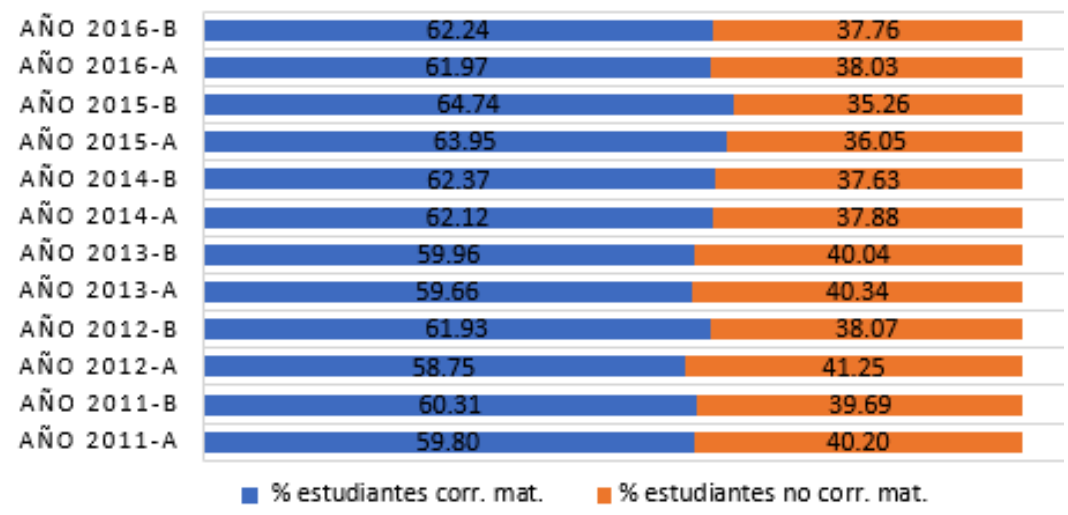

Figura 3. Porcentaje de estudiantes a los que corresponde matrícula regular y matrícula rezagada.

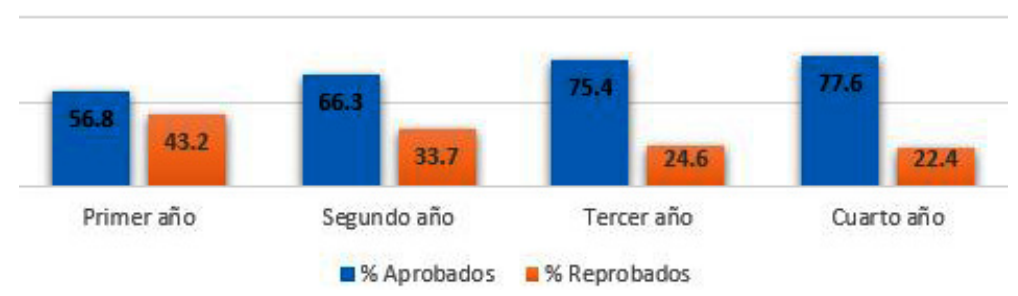

Figura 4. Porcentaje de estudiantes aprobados y reprobados.

y criterios asumidos en cualquier metodología de evaluación y acreditación de la calidad educativa, esencialmente para la calificación del estudiante, lo que requiere posteriormente un proceso de valoración.

Dado que la mayoría de estudiantes con asignaturas reprobadas se ubica en el primer año de estudios, se decidió caracterizar el rendimiento de los estudiantes en este año. Entre los indicadores cuantitativos del rendimiento, se trabajó con los detallados en la Tabla 2.
En la Tabla 3, se muestran los indicadores (promedio entre los años 2011-2016) calculados para las asignaturas "críticas" del primer año y que son causa de rezago en la matrícula. Los grupos tienden a ser numerosos, generalmente de 60 estudiantes, las calificaciones promedio obtenidas son muy bajas. En las asignaturas del primer semestre los índices de aprobación están alrededor del $30 \%$, la cantidad de estudiantes reprobados es muy alta, la deserción es cercana al 30\%, la repitencia (acción

Tabla 2. Conjunto de indicadores utilizados para describir el rendimiento académico.

\begin{tabular}{|c|c|c|c|c|c|}
\hline $\begin{array}{c}\text { Índice } \\
\text { aprobación }\end{array}$ & $\begin{array}{c}\text { Índice } \\
\text { reprobación }\end{array}$ & $\begin{array}{c}\text { Índice } \\
\text { deserción }\end{array}$ & $\begin{array}{c}\text { Índice } \\
\text { retención }\end{array}$ & $\begin{array}{c}\text { Índice } \\
\text { repetición }\end{array}$ & $\begin{array}{l}\text { Nivel dificultad/ } \\
\text { curso }\end{array}$ \\
\hline $\begin{array}{l}\text { Proporción de } \\
\text { estudiantes que } \\
\text { aprueban un curso y } \\
\text { que potencialmente } \\
\text { son aspirantes } \\
\text { a ingresar al } \\
\text { siguiente. }\end{array}$ & $\begin{array}{l}\text { Proporción de } \\
\text { estudiantes que } \\
\text { no aprueban el } \\
\text { curso en el cual } \\
\text { se inscribieron. }\end{array}$ & $\begin{array}{l}\text { Proporción de } \\
\text { estudiantes inscritos } \\
\text { en una determinada } \\
\text { asignatura, de un } \\
\text { determinado ciclo } \\
\text { que abandonan la } \\
\text { asignatura sin haber } \\
\text { culminado el ciclo. }\end{array}$ & $\begin{array}{l}\text { Proporción de estudiantes } \\
\text { inscritos en una } \\
\text { determinada asignatura, } \\
\text { de un determinado ciclo } \\
\text { que califican para pasar } \\
\text { a la siguiente asignatura } \\
\text { propuesta en el plan de } \\
\text { estudios vigente. }\end{array}$ & $\begin{array}{l}\text { Proporción de } \\
\text { estudiantes que } \\
\text { permanecen } \\
\text { en el mismo } \\
\text { curso durante } \\
\text { más de un ciclo } \\
\text { académico. }\end{array}$ & $\begin{array}{l}\text { Función de los } \\
\text { estudiantes que } \\
\text { no lograron } \\
\text { aprobar el curso. }\end{array}$ \\
\hline
\end{tabular}


Tabla 3. Indicadores del rendimiento académico promedio en el primer año de estudios.

\begin{tabular}{|l|r|c|c|c|c|c|c|c|}
\cline { 2 - 10 } \multicolumn{1}{l|}{} & $\mathbf{N}^{\mathbf{0}}$ est. & Media & Aprobación & Reprobación & Deserción & Retención & Repetición & $\begin{array}{c}\text { Nivel } \\
\text { dificultad }\end{array}$ \\
\hline Calculo en una variable & 183 & 6,6 & 35,1 & 38,0 & 26,9 & 73,1 & 48,7 & 64,9 \\
\hline Calculo en varias variables & 86 & 9,0 & 58,6 & 28,2 & 13,1 & 86,9 & 39,9 & 41,4 \\
\hline Estructuras discretas 1 & 189 & 6,6 & 31,3 & 40,5 & 28,2 & 71,8 & 45,8 & 68,7 \\
\hline Estructuras discretas 2 & 92 & 9,5 & 63,8 & 20,3 & 15,9 & 84,1 & 30,5 & 36,2 \\
\hline Fundamentos programación & 127 & 8,3 & 53,3 & 35,6 & 11,2 & 88,8 & 19,7 & 46,7 \\
\hline
\end{tabular}

de cursar reiteradamente una asignatura, sea por mal rendimiento del estudiante o por causas ajenas al ámbito académico) se aproxima al 50\% y el nivel de dificultad encontrado supera al $60 \%$. Estos indicadores mejoran ligeramente en el segundo semestre, pero en general revelan que el gran problema está en el primer año de estudios.

En la Figura 5, se grafica la relación entre el puntaje promedio, el índice de reprobación y el nivel de dificultad de las asignaturas mencionadas. De la gráfica se deduce que es necesario profundizar en la relación entre el nivel de dificultad y el índice de reprobación de las asignaturas.

La Figura 6 muestra los promedios ponderados de las calificaciones obtenidas en cada asignatura crítica entre los años 2013 y 2016, en ninguno de los casos se logra un promedio aprobatorio; sin embargo, es posible afirmar que en las asignaturas críticas en

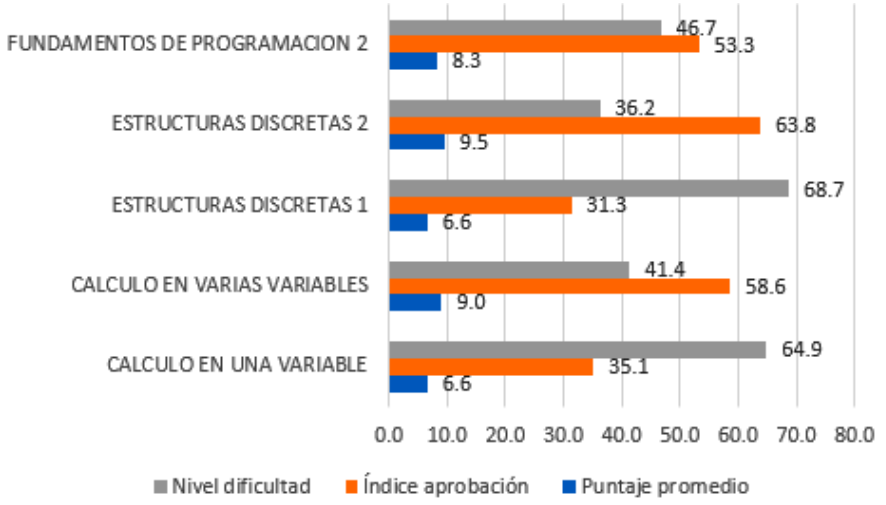

Figura 5. Relación entre el rendimiento promedio, el índice de reprobación y el nivel de dificultad de las asignaturas críticas de primer año.

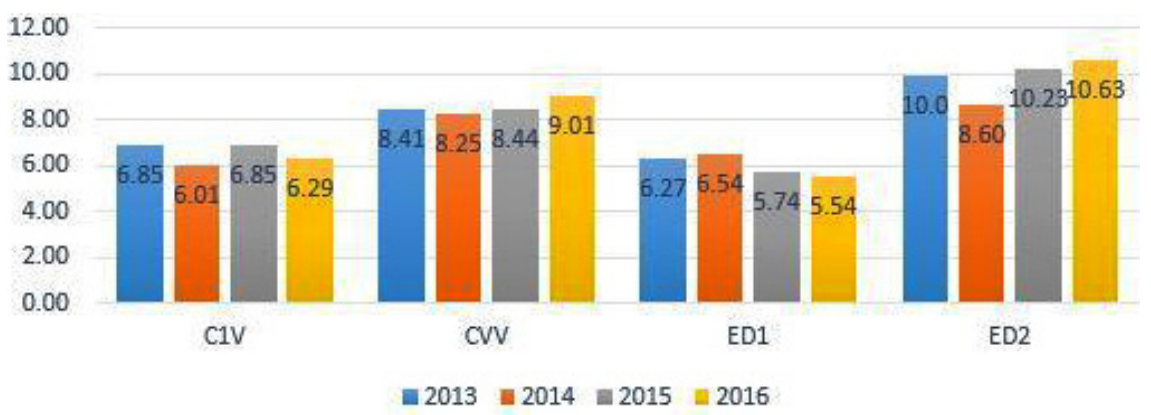

Figura 6. Calificaciones promedio obtenidas en las asignaturas críticas en los años 2013 al 2016. 
el año 2016 hubo mejor rendimiento promedio en comparación a los años anteriores.

\section{PROCESO DE MINADO DE DATOS}

En un proceso de Minería de Datos (MD), la utilidad del modelo depende principalmente de dos factores: la capacidad del modelo para descubrir patrones interesantes, y la facilidad con la cual la estructura del modelo puede ser entendida y ajustada por usuarios/analistas de datos [17].

Considerando que la minería de datos conjuga la estadística y las ciencias de la computación para intentar descubrir patrones en grandes volúmenes de datos, se propuso desarrollar un modelo que permita clasificar automáticamente a los estudiantes de EPISUNSA en función a su comportamiento académico, para ello se debía: (a) Identificar los diferentes factores que afectan el rendimiento académico del estudiante, (b) Probar diferentes modelos de predicción del comportamiento académico y (c) Proponer un modelo predictor del comportamiento académico.

Se ha utilizado la técnica de clasificación, que consiste en utilizar un conjunto pre-clasificado de ejemplos para desarrollar un modelo que permita clasificar un gran volumen de información. Esta técnica utiliza métodos de Árbol de decisión o Redes neuronales.

El proceso de clasificación de datos implica dos fases: el aprendizaje y la clasificación. En la fase de aprendizaje, mediante el algoritmo de clasificación, se analizan los datos de entrenamiento. El algoritmo de aprendizaje de clasificación usa los ejemplos pre-clasificados para determinar el conjunto de parámetros que se necesitan para realizar una discriminación adecuada. El algoritmo codifica estos parámetros en un modelo llamado clasificador. Luego el proceso de clasificación involucra un conjunto de entrenamiento consistente de datos con etiquetas de clase conocidas, conjunto que se usa para construir un modelo de clasificación que posteriormente se aplica a un conjunto de prueba que posee datos con etiquetas de clase desconocidas.

La fase de clasificación de los datos de prueba sirve para estimar la precisión de las reglas de clasificación. Si la precisión es aceptable, entonces las reglas se pueden aplicar a los datos.

\section{Técnicas, herramientas y metodología}

Sobre los datos provenientes de las cohortes 20112016, se han aplicado tres técnicas de minería de datos: Redes Neuronales, Árboles de decisión y Clustering. Se utilizó la herramienta IBM SPSS Modeler ya que proporciona algoritmos y modelos completos que están listos para su utilización inmediata, así mismo posee una interfaz integrada para ejecutar diferentes procesos. En el caso de los árboles de decisión, para la implementación de los algoritmos ID3 y C4.5 se implementó un aplicativo a medida desarrollado en JAVA.

Para el ciclo de vida de un proyecto de Data Mining, la metodología CRISP-DM propone seis fases, que interactúan entre ellas de forma iterativa durante su desarrollo.

1. Fase de análisis del problema (Comprensión del negocio).

2. Fase de análisis de datos (Comprensión de los datos).

3. Fase de preparación de datos.

4. Fase de modelado.

5. Fase de evaluación.

6. Fase de explotación o despliegue.

En lo relatado hasta el momento se han desarrollado los pasos relacionados con la comprensión del negocio y la comprensión de los datos. La fase de explotación no se ha considerado.

\section{Fase: Preparación de los datos}

Esta fase incluye varias tareas: selección de datos, limpieza de datos, generación de variables adicionales, integración de diferentes orígenes de datos y cambios de formato.

La data con que se cuenta está compuesta por 976 registros capturados en la ficha de matrícula al momento en que ingresan a la universidad, 584 registros recabados por la Oficina de Admisión y 26069 registros de calificaciones.

En ese orden de actividades, se omitieron aquellos registros con campos vacíos o que no lograban relacionarse, por ejemplo, un estudiante al que no se le relacionaba calificaciones. Como algunos registros pertenecían a años anteriores al 2011 se realizó el filtrado (nodo "Seleccionar") para obtener únicamente información de los periodos 2011 al 2016. 
Se integró en un solo archivo, las calificaciones por asignatura, los datos personales y el puntaje de ingreso de cada estudiante, para ello se utilizó como variable de enlace el CUI. En los casos que se consideró necesario también se hizo la conversión de datos alfanuméricos a datos numéricos.

A partir de los datos originales se seleccionaron como variables, aquellos que aportaban información a la investigación, con ellas se construyeron otras variables, las más importantes se describen a continuación:

- Promedio Ponderado Ajustado (PPA): Se obtiene al dividir la suma ajustada (créditos por la calificación final, en todas las matrículas registradas) entre el total de los créditos cursados.

- Índice de Rendimiento Exógeno (IREX): establece la relación entre el número de asignaturas aprobadas por el estudiante y el número de asignaturas aprobadas por el grupo.

- Índice de Rendimiento Endógeno (IREN): establece la relación entre el número de asignaturas aprobadas y el número de asignaturas matriculadas (considera además el número de asignaturas cursadas).

- Índice de Rendimiento Total (IRT): índice de rendimiento académico de cada estudiante, considera su desempeño y su comportamiento en relación al grupo.

\section{Fase: Modelado}

El descubrir conocimiento en bases de datos es un proceso complejo, según [18] muchos obstáculos, preguntas de investigación y problemas requieren ser investigados y clarificados.

Siguiendo la metodología CRISP-DM, se seleccionaron las técnicas de modelado más apropiadas para la investigación. La selección del modelo se realizó en base a los siguientes criterios: (a) Ser adecuado al problema, (b) Disponer de datos adecuados, (c) Cumplir los requisitos del problema, (d) Tiempo adecuado para obtener un modelo y

(e) Conocimiento de la técnica.

Atendiendo a estos criterios, se desarrollaron dos procesos uno con IBM SPSS Modeler y el otro con el aplicativo propio diseñado e implementado para estos fines.

\section{Proceso con IBM SPSS Modeler}

Se utilizó la funcionalidad de IBM SPSS Modeler, nodo "Clasificador automático" y se obtuvieron algunos resultados preliminares sobre las técnicas de minería de datos que mejor se ajustan a este problema. Para particionar la base de datos se utilizó el nodo "Partición", como resultado se particionó la data en dos conjuntos cuyos elementos se asignaron al azar. El conjunto de datos de entrenamiento contenía el $70 \%$ de la data original y el conjunto de prueba el $30 \%$ restante. La Figura 7, muestra los resultados.

\section{Proceso con el aplicativo propio}

Se ha implementado una aplicación que utiliza información proporcionada por la universidad y que genera modelos de clasificación a partir de diferentes algoritmos (redes neuronales, ID3, C4.5), y utiliza los atributos más significativos dentro de la información disponible.

Se particionó la base de datos en dos subconjuntos, se tomó aleatoriamente un $80 \%$ de los datos para formar el conjunto de datos de entrenamiento y se dejó el 20\% restante para el conjunto de prueba.

Con el conjunto de entrenamiento se entrenó los modelos de clasificación: redes neuronales (PERCEPTRÓN Multicapa) y árboles binarios (algoritmos C4.5 e ID3). Se evaluó durante este proceso el tiempo de entrenamiento que toma ejecutar cada uno de los algoritmos, esto se hizo en razón de que este puede ser un indicador significativo,

\begin{tabular}{|c|c|c|c|c|c|}
\hline ¿Utilizar? & Gráfico & Modelo & $\begin{array}{l}\text { Tiempo de generación } \\
\text { (min) }\end{array}$ & $\begin{array}{l}\text { Precisión } \\
\text { general (\%) }\end{array}$ & $\begin{array}{l}\text { Número de campos } \\
\text { utilizados }\end{array}$ \\
\hline 口 & & & $<1$ & 94,857 & 15 \\
\hline$\checkmark$ & & & $<1$ & 94,286 & 7 \\
\hline$\square$ & & salo & $<1$ & 93,714 & 5 \\
\hline
\end{tabular}

Figura 7. Tabla de resultados de la clasificación automática. 
pues si se desea cambiar de base de datos, el tiempo puede variar significativamente debido al número de variables de entrada y al tamaño del conjunto de entrenamiento. Para realizar el entrenamiento de los modelos se le proporcionó al sistema un archivo con las variables de entrada y un campo clasificado como salida (abandona o no) del modelo. También se le proporcionó un archivo con la lista de los códigos de estudiantes que componían el conjunto de entrenamiento junto con la etiqueta de clase a la que pertenecían, clasificación que se hizo previamente en base a información real (Tabla 4).

Esta acción se justifica en que el entrenamiento se realiza usando datos conocidos y cuando se realiza la clasificación real, se hace sobre datos no trabajados ni en la fase de entrenamiento ni en la fase de prueba, por lo que los resultados así obtenidos son una predicción. El sistema recibe una matriz (Estudiante x Variables) y construye, según la elección del usuario, un modelo de clasificación.

Para la opción red neuronal, se construyó una red perceptrón multicapa entrenada con el algoritmo backpropagation. Para detener el entrenamiento, se establecieron dos criterios diferentes: llegar a una tasa de error del $0,01 \%$ o llegar a un máximo de 100000 iteraciones. Al terminar el entrenamiento, se guardaron los pesos de las conexiones de las neuronas en un archivo, lo que permitiría armar nuevamente la red en caso se quisiera utilizar nuevamente el modelo.

Luego de la fase de entrenamiento de los algoritmos ID3 y C4.5 (algoritmos que construyen un árbol de decisión utilizando fórmulas de entropía y ganancia de información), se construyeron los árboles de decisión respectivos y se guardaron en la base de datos del sistema. En el caso del algoritmo C4.5 se hizo uso de las fórmulas de la tasa de ganancia (Gain Ratio) en lugar la ganancia; y la fórmula de información de división.

En cualquiera de los tres modelos explicados, las variables de salida clasifican los registros en NO ABANDONA y ABANDONA.

\section{FASE: EVALUACIÓN Y DISCUSIÓN DE LOS RESULTADOS}

\section{Proceso con IBM SPSS Modeler}

Las técnicas de clasificación que mejor precisión obtuvieron a partir de la ejecución del Nodo "Clasificador automático" fueron:

- Árboles Aleatorios: Según la matriz de confusión existen 595 registros correctamente clasificados y 8 registros erróneos. Se obtuvo un $94,86 \%$ de precisión y $5,14 \%$ de error.

- QUEST 1: Según la matriz de confusión existen 381 registros correctamente clasificados y 22 registros erróneos. Se obtuvo $94,29 \%$ de precisión y $5,71 \%$ de error.

- CHAID 1: Según la matriz de confusión existen 387 registros correctamente clasificados y 16 registros erróneos. Se obtuvo 93,71\% de precisión y $6,29 \%$ de error.

La técnica de árboles aleatorios es la que produjo métricas de rendimiento con mejores resultados, resultando que el índice de rendimiento exógeno (con importancia 0,12 ) es la más significativa en la predicción del índice de rendimiento total (IRT).

De acuerdo a la literatura existente se han categorizado los valores del IRT y del PPA, las categorías se muestran en la Tabla 5.

Tabla 4. Segmento del conjunto de prueba.

\begin{tabular}{|l|c|c|l|c|l|}
\hline \multicolumn{1}{|c|}{ Género } & Promedio No... & Relación Cr... & Colegio Pro... & Puntaje Exa... & Clasificación \\
\hline Masculino & 11,0 & 73,0 & Parroquial & 53,0 & No Abandona \\
\hline Masculino & 3,0 & 10,0 & Parroquial & 63,0 & Abandona \\
\hline Masculino & 6,0 & 10,0 & Estatal & 00,0 & Abandona \\
\hline Masculino & 10,0 & 38,0 & Parroquial & 58,0 & Abandona \\
\hline Femenino & 9,0 & 47,0 & Particular & 48,0 & No Abandona \\
\hline Femenino & 10,0 & 53,0 & Parroquial & 56,0 & Abandona \\
\hline Masculino & 13,0 & 86,0 & Parroquial & 68,0 & No Abandona \\
\hline Femenino & 10,0 & 62,0 & Particular & 48,0 & No Abandona \\
\hline
\end{tabular}


Tabla 5. Categorización del IRT y del PPA.

\begin{tabular}{|c|l|l|l|c|}
\hline IRT & \multicolumn{1}{|c|}{ Caso } & \multicolumn{1}{|c|}{ Categoría } & \multicolumn{1}{c|}{ Caso } & PPA \\
\hline 5 & Más del $80 \%$ & Sobresaliente & Más de 17 puntos & 5 \\
\hline 4 & Entre 65 y 80\% & Muy bueno & Entre 15 y 17 puntos & 4 \\
\hline 3 & Entre 50\% y 64\% & Bueno & Entre 13 y 14 puntos & 3 \\
\hline 2 & Entre 34\% y 49\% & Suficiente & Entre 11 y 12 puntos & 2 \\
\hline 1 & Entre 15\% y 33\% & Bajo & Entre 8 y 10 puntos & 1 \\
\hline 0 & Menos del 15\% & Muy bajo & Menos de 8 puntos & 0 \\
\hline
\end{tabular}

Considerando el Índice de Rendimiento Total (IRT) se pueden identificar características con respecto al comportamiento académico de los estudiantes:

- El mayor porcentaje de mujeres (52\%) cuenta con un IRT = 2 ("SUFICIENTE"), mientras que el mayor porcentaje de hombres $(43 \%)$ cuenta con IRT $=3$ ("BUENO")

- Los estudiantes con IRT = 4 ("MUY BUENO") pertenecen a alumnos cuyas edades al momento de ingresar a la universidad estaban entre los 17 y 18 años

- Los estudiantes clasificados con IRT $=4$ ("MUY BUENO") son aquellos con Promedio Ponderado Ajustado $=3$ (Notas entre 13 y 14)

- Los estudiantes con IRT = 4 ("BUENO") son quienes tienen Promedio Ponderado Ajustado $=2($ Notas entre 11 y 12$)$

- La mayoría de estudiantes con IRT = 4 ("MUY BUENO") obtuvo entre 50 y 70 puntos (sobre 100) en la prueba de admisión a la universidad.

- $\quad$ La cantidad de estudiantes con IRT = 0 ("MUY BAJO") e IRT = 5 (“SOBRESALIENTE”) fue mínima, por lo que no resultó significativa.

De la clasificación resultó que, considerando el IRT, los estudiantes se clasifican mayormente en las categorías "Suficiente" (41\%) y "Bueno" (40-48\%). Solo el 6\% de los estudiantes alcanza la categoría "Muy bueno".

Al considerar el PPA, la clasificación cambia, se puede enunciar que cerca de la mitad de los estudiantes $(47,58 \%)$ se clasifica en la categoría "Muy bajo", y que la mayoría de estudiantes (92\%) consigue un promedio de calificaciones menor o igual a 12 puntos.

Al clasificar a los estudiantes según el IRT, en la categoría "Suficiente" se mantiene la proporción entre el número de estudiantes varones y el de mujeres; sin embargo, en la categoría "Bueno", esa relación se rompe y la proporción resulta casi igual. De lo observado se deduce que hay mayor cantidad de estudiantes mujeres con buenos resultados académicos.

Al considerar el índice de Rendimiento Total, los estudiantes se clasifican mayormente en las categorías "Suficiente" (41\%) y "Bueno" (40-48\%). Solo el $6 \%$ de los estudiantes alcanza la categoría "Muy bueno". Al considerar el Promedio Ponderado Ajustado, la clasificación cambia, se puede enunciar que cerca de la mitad de los estudiantes $(47,58 \%)$ se clasifica en la categoría "Muy bajo", y que la mayoría de estudiantes (92\%) consigue un promedio de calificaciones menor o igual a 12 puntos. La situación descrita, lleva a reflexionar sobre las características de la evaluación de los resultados de aprendizaje de los estudiantes.

\section{Proceso con el aplicativo propio}

Al evaluar los resultados con cada una de las técnicas utilizadas se observa:

- C45: Se obtuvo un 68\% de exactitud (qué tan cerca está un valor medido respecto al valor verdadero), un $65 \%$ de precisión (proporción de casos que realmente tiene la clase real) y un $92 \%$ de sensibilidad.

- Red Neuronal: Se dieron resultados muy similares con medidas de rendimiento del $66 \%, 62 \%$ y $85 \%$ de exactitud, precisión y sensibilidad respectivamente.

- ID3: Produjo resultados de 60\%, 58\% y $75 \%$ respectivamente.

El algoritmo de C4.5 es el que produjo métricas de rendimiento con mejores resultados, siendo la variable más significativa la relación de créditos aprobados por un estudiante en relación a los créditos teóricos que debería haber aprobado. 
La Figura 7 muestra el árbol de decisión construido por el clasificador C.45.

Un árbol de decisión puede representarse linealmente por medio de reglas de decisión, donde el resultado es el contenido del nodo hoja, y las condiciones a lo largo del camino forman una conjunción con la cláusula "if", entonces el árbol obtenido se podría interpretar el árbol de la siguiente manera:

- Si la RELACIÓN DE CRÉDITOS (créditos actuales/créditos que debería tener) es menor o igual al $40 \%$ entonces el estudiante corre el riesgo de abandonar.

- Caso contrario, si su PUNTAJE EN EL EXAMEN DE ADMISIÓN fue menor o igual a 46 entonces corre el riesgo de abandonar.

- Caso contrario, si el PROMEDIO DE NOTAS es menor a 7 entonces corre el riesgo de abandonar.

- Caso contrario, si el GÉNERO del estudiante es mujer no abandona la carrera. Si es hombre entonces abandonará la carrera si ha estudiado en un colegio particular y no abandona si ha estudiado en un colegio estatal o parroquial.

Los resultados muestran que el algoritmo de C4.5 presentó mejoras medidas de rendimiento con respecto a la red neuronal y al ID3 y que la relación de créditos aprobados por un estudiante con respecto a los créditos que debería haber llevado es la variable más significativa en la construcción del modelo, seguida por las calificaciones, mientras que, la modalidad de examen de admisión mediante la cual el estudiante ingresó a la universidad no resultó ser significativa, pues no aparece en el árbol de decisión generado.

\section{PROPUESTA DE MEDIDAS REMEDIALES}

Generalmente al hacer referencia a comportamiento académico se alude a un sistema de calificaciones, sin embargo, los expertos en evaluación afirman que no es suficiente un proceso de calificación, debe integrarse un proceso de evaluación, es decir, un proceso que lleve a formular un juicio de valor que a la valoración de los conocimientos adquiridos integre valores, habilidades, comportamientos y desempeño del estudiante en todas las actividades académicas. Un acercamiento a medir el rendimiento académico de un estudiante en una asignatura, en un semestre, en un año lectivo, debe ser la suma ponderada de las calificaciones obtenidas en los exámenes parciales, más las calificaciones obtenidas en otras actividades académicas como controles de lectura, evaluaciones cortas, monografías, trabajos de campo, laboratorios, actividades de investigación formativa, proyectos de intervención social, etc. Esta suma resultaría en la calificación final obtenida por el estudiante y representaría su rendimiento.

Las Teorías Curriculares, Pedagógicas y Didácticas son ciencias que indiscutiblemente precisan de toda la información que ofrecen las técnicas antes descritas, que van desde el análisis de datos para evitar la reprobación y la deserción estudiantil hasta el descubrimiento de patrones de comportamiento. En [19] se menciona que "llega un momento en el que

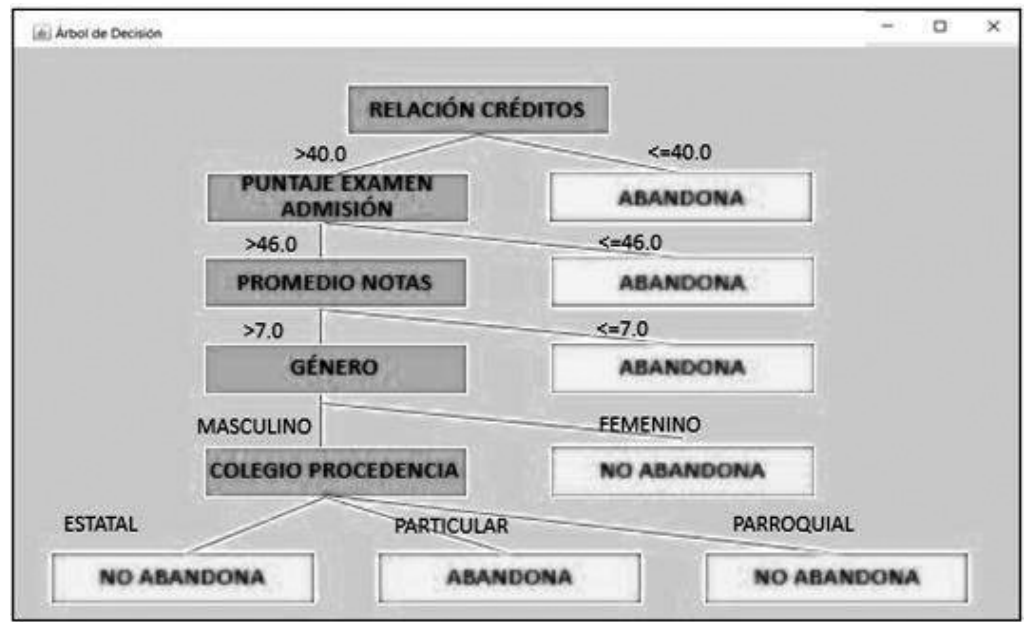

Figura 8. Árbol de Decisión Generado por el Aplicativo. 
la acumulación de pequeños ajustes en las formas culturales para aprender y enseñar ya no es suficiente y se necesita una verdadera reestructuración, un cambio radical de las estructuras y hábitos".

Es por ello que, considerando los resultados presentados, la literatura consultada y la experiencia de los investigadores que forman parte del proyecto se proponen algunas alternativas de conducentes a aminorar los efectos del rezago y el abandono estudiantil:

1. Prestar mayor atención a las asignaturas consideradas "críticas", en ellas resulta fundamental la actualización didáctica de los profesores que las imparten. Es importante mostrar la aplicabilidad de estas asignaturas en el desempeño profesional del estudiante en formación, pero lo es más aún, que el profesor utilice métodos, técnicas y medios que propicien la participación del estudiante como centro del proceso de formación.

2. La integración de técnicas cooperativas, gamificación, procesos de aula invertida, prácticas de laboratorio virtual, convenientemente soportadas en el uso de plataformas educativas virtuales, videos educativos y metodologías participativas en los procesos de enseñanzaaprendizaje aumenta la motivación, el interés y por tanto los resultados de aprendizaje de los estudiantes [20-21].

3. Sería ideal que las asignaturas críticas estuvieran a cargo de uno o más profesores con experiencia profesional y con calidad didáctica, de modo que puedan orientar el proceso de enseñanzaaprendizaje desde sus aportes a la temática en estudio y al mismo tiempo hacer seguimiento al aprendizaje.

4. La dificultad observada, de aprobar ciertas asignaturas, debe llevar a analizar la estructura dentro del plan de estudios de estas asignaturas, de modo que no se cargue en demasía el trabajo del estudiante y se pueda definir una secuencia de actividades que facilite el tránsito por estas asignaturas. Así mismo, se debe normar un número máximo de estudiantes permitidos en cada asignatura (o en los grupos de una misma asignatura).

5. Es urgente implementar acciones de seguimiento y atención a las particularidades de los estudiantes, específicamente en el caso del género femenino, mayormente afectado por la deserción y abandono escolar. Se sugiere asignar asesores académicos o ayudantes de cátedra que tomen la responsabilidad de acompañar en el aprendizaje a los estudiantes que han sido clasificados como estudiantes en riesgo académico.

6. Es necesario implementar alguna medida con el fin de allanar la diferencia entre los conocimientos y habilidades prerrequisito, necesarios para desarrollarse en la universidad, y los que el estudiante ha desarrollado en la educación básica. En particular, se puede afirmar que los estudiantes que egresan de la educación básica no reúnen ni el conocimiento ni las competencias matemáticas necesarias para lograr un buen desempeño académico en su trayectoria universitaria. Se sugiere la implementación de un ciclo cero o ciclo de nivelación.

\section{CONCLUSIONES}

Del análisis de la matrícula considerando la relación año de ingreso-año cronológico, se desprende que una medida del rendimiento académico sería la relación entre el número de créditos aprobados y el número de créditos en los que un estudiante registró matrícula.

Así mismo, los resultados muestran que en el rendimiento total influye la relación entre el número de asignaturas aprobadas por el estudiante y el número de asignaturas aprobadas por el grupo. Luego, a la hora de analizar el rendimiento académico de un estudiante es importante ir más allá de las calificaciones obtenidas y considerar su comportamiento académico, su rendimiento en relación a su cohorte y el ritmo de avance en la aprobación de las asignaturas.

Las técnicas empleadas en esta investigación han sido útiles para entender algunas características del comportamiento académico y obtener modelos predictivos que permitan identificar oportunamente a los estudiantes en riesgo académico.

Las propuestas para la retención de los estudiantes requieren ser contextualizadas y atendidas individualmente, de modo que se pueda observar su desarrollo y evolución.

Finalmente, toda la información que permita relacionar a los estudiantes con los resultados de los 
procesos de enseñanza-aprendizaje puede facilitar los resultados educativos esperados y optimizar las potencialidades de las técnicas, métodos, herramientas y alternativas para su perfeccionamiento.

La educación universitaria precisa de toda la información posible para el cumplimiento de su misión social: formar profesionales a la altura de su tiempo y que respondan a las necesidades de su realidad.

\section{TRABAJOS FUTUROS}

El modelo puede mejorarse si se utiliza un mayor número de variables, por ejemplo, las relacionadas con factores socio-económicos.

Así mismo se propone.

\section{AGRADECIMIENTOS}

Expresamos el agradecimiento de las autoras a la Universidad Nacional de San Agustín de Arequipa por el apoyo recibido a la realización de la propuesta y esperamos que los resultados y reflexiones expresadas permitan que nuestra casa de estudios pueda tomar medidas que lleven a mejorar la calidad académica y personal de sus egresados y promover la permanencia de sus estudiantes.

\section{REFERENCIAS}

[1] I. Mushtaq and S. Nawaz. "Factors Affecting Students" Academic Performance". Mohammad Ali Jinnah University Islamabad, Pakistan. 2012.

[2] IESALC. "Informe sobre la educación superior en América latina y el caribe 2000-2005". Instituto Internacional de la UNESCO para la Educación Superior en América Latina y el Caribe. ISBN -980-6556-19-4. 2007.

[3] M. Ferreyra, C. Avitabile y J. Botero. "Momento decisivo: La educación superior en América Latina y el Caribe". Banco Internacional de Reconstrucción y Fomento/Banco Mundial. 2017. DOI: 10.1596/978-1-4648-1014-5.

[4] J. Fullana. "Revisió de la recerca educativa sobre les variables explicatives del rendiment acadèmic: Apunts per a l'ús del criteri de modificabilitat pedagógica de les variables". Estudi General. Vol. 12, pp. 185-200. 1992.
[5] J. Tourón. "Factores del rendimiento académico en la universidad". España: Ediciones Universidad de Navarra, S.A. 1984.

[6] M. Page. "Hacia un modelo causal del rendimiento académico". Madrid: Centro de publicaciones - Secretaría General Técnica, Ministerio de Educación y Ciencia. 1990.

[7] M. Benítez, M. Giménez y R. Osicka. "Las asignaturas pendientes y el rendimiento académico: ¿existe alguna relación?”. 2000.

[8] A. Pace. "Hacia un modelo causal del rendimiento académico". Investigación. Centro de Investigación y Documentación Educativa CIDE. Vol. 43. Madrid, España. 1990.

[9] J. Caso y L. Hernández. "Variables que inciden en el rendimiento académico de adolescentes mexicanos". Revista Latinoamericana de Psicología. Vol. 39 Nº 3, pp. 487-501. 2007.

[10] V. Tinto. "Definir la deserción: una cuestión de perspectiva". Revista de Educación Superior. Vol. 71. ANUIES, México. 1989.

[11] V. Tinto. "Leaving College: Rethinking the Causes and Cures of Student Attrition". Chicago: The University of Chicago Press. 1987.

[12] J. Vásquez, E. Castaño, S. Gallón y K. Gómez. "Determinantes de la Deserción Estudiantil en la Universidad de Antioquia”. Universidad de Antioquia, Facultad de Ciencias Económicas. Medellín: Universidad de Antioquia. 2003.

[13] E. Solano. "Causas e Indicadores de la Deserción en el Programa de Economía de la Universidad del Atlántico Aplicando Modelos de Duración y Microeconómico". Universidad del Atlántico, Barranquilla. 2006.

[14] M. Rodríguez y M. Ruiz. "Indicadores de rendimiento de estudiantes universitarios versus créditos acumulados". Revista de educación, pp. 467-492. 2011.

[15] L. Vega. "Modelo Educativo para el Siglo XXI: formación y desarrollo de competencias profesionales". México: Secretaría de Educación Pública, Dirección General de Educación Superior Tecnológica. 2012.

[16] A. Torres y Z. Lima. "Criterios cuantitativos de eficiencia pedagógica en la formación del profesional de Agronomía". Revista Pedagogía Universitaria. Vol. 8 Nº 5. 2003. 
[17] W. Castillo-Rojas, A. Peralta and C. Vargas. "Exploratory and interactive visualization model for association rules". Ingeniare. Revista chilena de ingeniería. Vol. $23 \mathrm{~N}^{\circ} 4$, pp. 505-513. 2015.

[18] W. Castillo-Rojas, F. Medina-Quispe and C. Meneses-Villegas. "Augmented decision tree models using self-organizing maps". Ingeniare. Revista chilena de ingeniería. Vol. $22 \mathrm{~N}^{\circ}$ 3, pp. 351-362. 2014.

[19] C. Monereo y J. Pozo. "Competencias para convivir con el siglo XXI”. En C. Monereo y J.I. Pozo, (Coords.) Monográfico sobre competencias básicas. Cuadernos de Pedagogía No 370, pp. 12-17. 2007.
[20] N. Bedregal. "Cooperative learning using Moodle as a support resource: Proposal for continuous evaluation in operational research". Proceedings - International Conference of the Chilean Computer Science Society, SCCC. Vol. 2017-October, pp. 1-9. July 5, 2018. DOI: 10.1109/SCCC.2017.8405131.

[21] N. Bedregal and D. Tupacyupanqui. "Integration of active methodologies and virtual classroom in the teaching-learning processes of Discrete Mathematics". Proceedings of the LACCEI international Multi-conference for Engineering, Education and Technology. Vol. 2018-July. 2018. DOI: 10.18687/LACCEI2018.1.1.81. 